\title{
The management of acute ischemic stroke - a role for percutaneous intervention?
}

\section{Mark Abelson}

Vergelegen Medi-clinic, Somerset West

Address for correspondence:

Dr Mark Abelson

PO Box 2543

Somerset West

7129

South Africa

Email:

mark@helderbergheart.co.za

\section{ABSTRACT Acute ischemic stroke due to large vessel occlusion is a devastating disease. In the vast majority of cases little is done to reverse the actual cause, and that is to open the occluded artery. This article reviews the current standard management with intra- venous thrombolysis and highlights some of its limitations, and the more recent development of mechanical embolectomy for the emergency treatment of ischemic stroke. SAHeart 2008; 5: 102-105}

\section{INTRODUCTION}

Worldwide stroke is a leading cause of death and disability and was recently declared a national disaster in South Africa. (I) Stroke management depends on the type of stroke (ischemic or hemorrhagic), its size (large or small artery involved), and the time of presentation after onset. The majority of strokes (approximately 85\%) are ischemic in nature and therefore may be amenable to reperfusion therapy. As with ST elevation myocardial infarction (STEMI) patients, early reperfusion should be the primary therapeutic strategy for ischemic stroke. However, the therapeutic window for intravenous (iv) thrombolysis is very narrow, being less than 3 hours after stroke onset. ${ }^{(2)}$ This window can be extended for up to 6 hours for intra-arterial (ia) thrombolysis. ${ }^{(3,4)}$ and for up to 8 hours for mechanical embolectomy. ${ }^{(5)}$
It is important to appreciate that there are key pathological differences between STEMI and ischemic stroke. STEMI is usually the result of plaque rupture and in-situ thrombosis, whereas ischemic stroke most often results from athero-thrombotic embolism. ${ }^{(6)}$

The outcome of large strokes is very poor. The reported mortality for untreated terminal occlusion of the internal carotid artery (ICA) is 53\% and up to $90 \%$ for basilar artery occlusion. The vast majority of those who survive are left with severe neurological disability.

\section{STROKE : INITIAL EVALUATION}

Initial evaluation involves assessing the clinical deficit according to the National Institute of Health Stroke Scale (NIHSS) (www.ninds.nih.gov/ doctors/NIH_Stroke_Scale.pdf). This score (0-42 with 0 being normal) is used to assess stroke severity and to help guide management decisions. Strokes can be divided into mild (NIHSS score $<7$ ), moderate (8-16), severe (17-24) and very severe (>24). Urgent brain imaging either by $\mathrm{CT}$ scanning or MRI is mandatory. The primary purpose of the initial scan is to rule out a hemorrhagic stroke, which is managed differently. More sophisticated MR perfusion-diffusion or CT perfusion scans can be obtained to assess the size of the ischemic but still viable brain tissue surrounding the infarcted area (ischemic penumbra). This may help to predict the response to reperfusion therapy. However, these scans are more expensive and, more importantly, time consuming. Current data suggests that the majority of strokes will have a significant ischemic penumbra within the first 6 to 8 hours of stroke onset and may well benefit from reperfusion therapy. ${ }^{(7,15)}$

\section{INTRAVENOUS THROMBOLYSIS}

Intravenous tissue Plasminogen Activator (tPA) $(0.9 \mathrm{mg} / \mathrm{kg}$, maximum dose $90 \mathrm{mg}$ ) carries the strongest recommendation for patients who present within 3 hours of ischemic stroke onset (Class I; Level of Evidence A). ${ }^{(7)}$ In the United States, however, only $2 \%$ of all ischemic stroke patients actually get this therapy, ${ }^{(6)}$ mainly because patients present beyond the 3-hour cut off. In the landmark NINDS Trial (2) comparing iv tPA to placebo in patients presenting within 3 hours of onset (median NIHSS score 14), $21 \%$ of the placebo group vs. $34 \%$ of 
the tPA group achieved full recovery at 3 months $(P<0.05)$. However, there was no overall mortality difference. The intracerebral bleeding rate was $6.4 \%$ with tPA and $0.6 \%$ in placebo treated patients.

Intravenous tPA has limited efficacy. In a study using transcranial Doppler I hour following iv tPA given within 3 hours of stroke onset, complete vessel patency is obtained in only 17\% of cases, with partial flow in $28 \%$ and no recanalization in over half the cases. ${ }^{(9)}$ The efficacy of iv tPA also depends on the site of arterial occlusion. Patency rates following iv tPA for terminal internal carotid artery (ICA) occlusion is less than 10\%. It is approximately 30\% for basilar and proximal middle cerebral artery (MCA) occlusions and 50\% for mid MCA occlusions. ${ }^{(10)}$ One-year outcome data from the NINDS trial(14) showed no benefit of iv tPA in those patients with a baseline very large stroke (NIHSS score $>20$ ).

\section{CATHETER-BASED THERAPY}

\section{Intra-arterial Thrombolysis}

The intra-arterial approach has been promoted because a high concentration of thrombolytic agent may be delivered into the thrombus. Despite the uncontrolled observation that recanalization rates may be higher with ia thrombolysis than with iv thrombolysis, clinical benefit may be counterbalanced by delays to initiating treatment with the ia approach.(7)

Intra-arterial administration of at least one specific thrombolytic agent, recombinant prourokinase, appears to be of some benefit in treatment of carefully selected patients with acute ischemic stroke secondary to occlusion of the MCA.(3) This conclusion was based on the results of a prospective, randomized, placebo-controlled phase III study testing the effectiveness of ia thrombolysis with prourokinase among patients with stroke of $<6$ hours' duration secondary to occlusion of the MCA. In the primary intent-to-treat analysis, $40 \%$ of the 121 patients treated with recombinant prourokinase and $25 \%$ of the 59 control patients had a modified Rankin Scale score of 0 to 2 at 90 days $(P=0.04)$. Recanalization of the MCA was achieved in $66 \%$ of the patients treated with recombinant prourokinase and $18 \%$ of the patients in the control group $(\mathrm{P}<0.00 \mathrm{I})$. Intracranial hemorrhage with neurological deterioration within 24 hours of treatment occurred in 10\% of patients treated with recombinant prourokinase and in $2 \%$ of the control group $(\mathrm{P}=0.06)$. No difference in overall death rate was seen between the 2 groups. The FDA has not approved the drug, and recombinant prourokinase is not currently available for clinical use. Extrapolation to tPA, the widely used iv cerebral thrombolytic drug, and to urokinase, which is chemically similar to the prourokinase prodrug, is based on consensus and case series data. Intra-arterial tPA has also been studied in combination with lower dose iv therapy $(0.6 \mathrm{mg} / \mathrm{kg})$ with promising results warranting further study. ${ }^{(4)}$

\section{Mechanical Embolectomy}

Mechanical embolectomy using the FDA approved Merci Retriever (Concentric Medical Mountain View, California, USA) is a new device used to extract the offending thrombo-embolus from large intracerebral vessels up to 8 hours post stroke onset. ${ }^{(5)}$ It may also be used safely in a combination with either iv or ia tPA.(I)

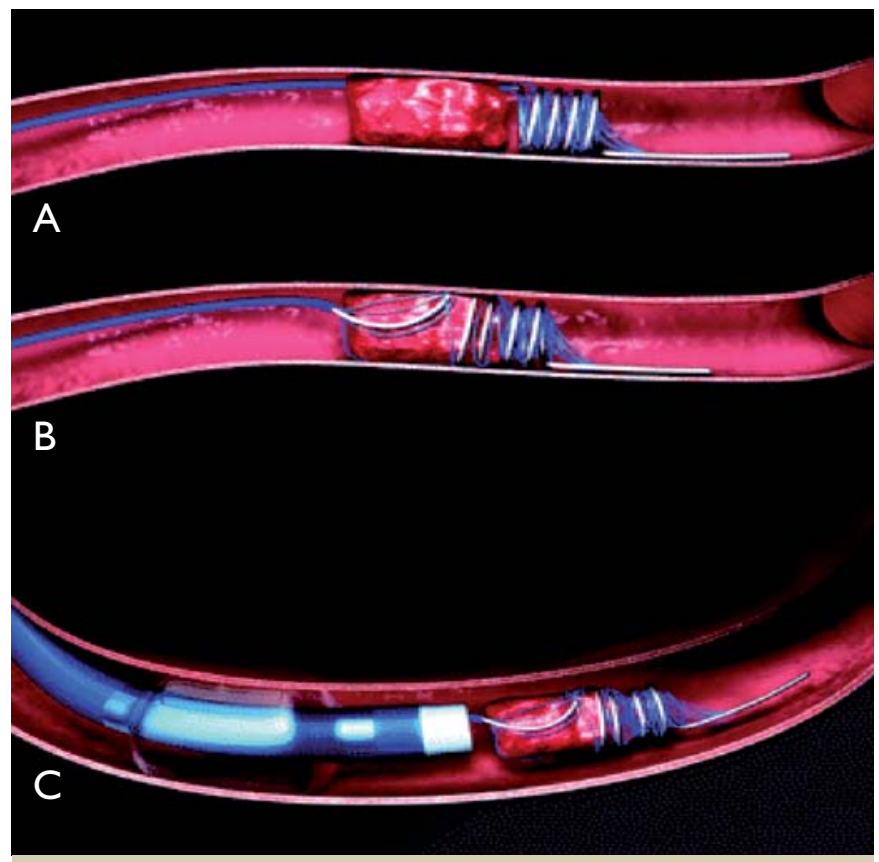

FIGURE I: Illustration of the Merci L5 thrombectomy device removing thrombus. The $L 5$ device is a helix of flexible nitinol wire with an arcade of filaments secured to the loops of the helix. This differs from the $X 5$ and X6 Merci Retrievers by having filaments and no taper to the coils. Within 8 hours of acute ischemic stroke, the balloon guide catheter is placed via femoral artery into the proximal internal carotid or vertebral artery. The blue microcatheter is advanced through the balloon guide catheter and placed through the occlusion using a microguidewire. The guidewire is then exchanged for the Retriever, which is advanced distal to the clot and several loops are deployed (A). The device is further deployed so as to fully ensnare the clot (B). Then, the proximal balloon of the guide is inflated to prevent distal embolization, some torquing maneuvers are applied, and the microcatheter and Retriever are withdrawn together to retrieve the clot (C).

(Reproduced with permission of Concentric Medical, Mountain View, California, USA)

The Merci(5) and Multi Merci trials ${ }^{(1)}$ were prospective, nonrandomized, multi-center trials which investigated the safety and efficacy of a novel embolectomy device (Merci Retriever) to open occluded large intracranial vessels (ICA, proximal MCA, Basilar artery) up to 8 hours after stroke onset. In the Merci trial all patients $(n=151)$ were ineligible for thrombolysis. In the Multi Merci trial patients $(n=164)$ were either ineligible for thrombolysis or had failed thrombolysis. Median baseline $\mathrm{NIHSS}=19$. A vessel patency rate of $57.3 \%$ with the device alone and $69.5 \%$ when combined with iv or ia thrombolytic was achieved.(II) Revascularization correlated with a good clinical outcome (Modified 
PERCUTANEOUS

INTERVENTION IN

ACUTE STROKE

Rankin Score <2 at 90 days) in $49 \%$ of those patients who were successfully revascularised compared to just $9.6 \%$ where revascularization failed $(\mathrm{p}<0.00 \mathrm{I})$. Mortality at 90 days was $25 \%$ against $52 \%$ respectively $(p<0.00 \mathrm{I})$ The rate of symptomatic intracerebral hemorrhage was $9.8 \%$ in the Multi Merci trial; however, only $2.4 \%$ were sizable enough to cause mass effect. ${ }^{(1)}$ This compared to $6.4 \%$ with iv tPA alone in the NINDS trial.(2) With growing experience and subtle device and technique modifications vessel patency rates of up to $83.6 \%$ are obtainable..$^{(12)}$

Mechanical embolectomy is undergoing further analysis in the MR Rescue and IMS-2 trials. Both trials are randomized and will provide important efficacy data with primary clinical outcomes.

In a recent meta-analysis on the impact of recanalization (spontaneous, post thrombolytic or mechanical) on ischemic stroke outcome, ${ }^{(12)}$ clinical outcome at 3 months is strongly related to successful reperfusion (odds ratio 4.43. $\mathrm{p}<0.000 \mathrm{I}$ ). Three month mortality was significantly reduced in recanalized patients (odds ratio 0.24. $p<0.000 \mathrm{I}$ ). Rates of symptomatic hemorrhagic transformation did not differ between the two groups (Figure 2).

To date, the author has used the Merci Retriever in six cases (Table I). Average age of 65 years, symptom duration of 4.2 hours and NIHSS score $=32$. In one case with a proximal middle cerebral artery occlusion it was not possible to deploy the device due to extensive vessel tortuosity. The patient was given ia tPA alone but failed to re-canalize and she suffered a large stroke. The device was successfully deployed

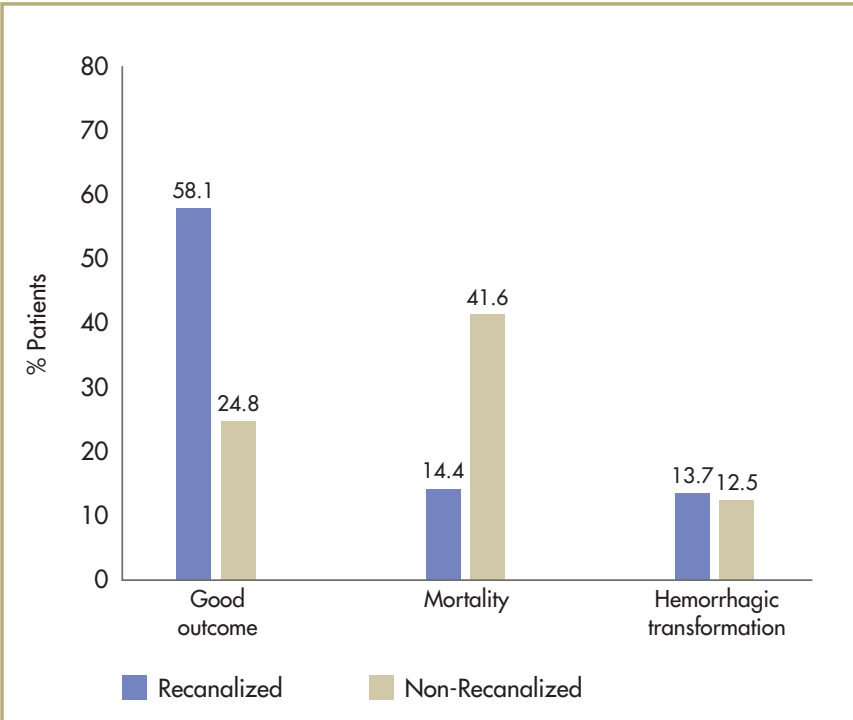

FIGURE 2: The impact of recanalization on ischemic stroke outcome: a meta analysis of 53 studies involving 2066 patients. ${ }^{(12)}$ Endpoints assessed were clinical outcome, mortality and hemorrhagic transformation of the infarct. (Modifed from Rha et al, Stroke 2007;38:967-973)
TABLE I: Patients treated by the author with the Merci Retriever

\begin{tabular}{|c|c|c|c|c|c|c|}
\hline Age & Gender & $\begin{array}{l}\text { Vessel } \\
\text { involved }\end{array}$ & $\begin{array}{l}\text { Symptom } \\
\text { duration } \\
(\mathrm{hr})\end{array}$ & $\begin{array}{l}\text { NIHSS } \\
\text { score }\end{array}$ & Reperfusion & Outcome \\
\hline 78 & $\mathrm{~F}$ & $\begin{array}{l}\text { Right ICA* } \\
\text { I00\% occlusion }\end{array}$ & 4 & 37 & $\begin{array}{l}\text { Complete MCA, } \\
\text { partial } \mathrm{ACA}^{\circ}\end{array}$ & Died \\
\hline 51 & $M$ & $\begin{array}{l}\text { Left ICA } \\
100 \% \text { occlusion }\end{array}$ & 3 & 32 & Complete & MRS 2 \\
\hline 66 & $\mathrm{~F}$ & $\begin{array}{l}\text { Left ICA } \\
100 \% \text { occlusion }\end{array}$ & 7 & 30 & Complete & MRS 3 \\
\hline 70 & $\mathrm{~F}$ & $\begin{array}{l}\text { Left ICA } \\
100 \% \text { occlusion }\end{array}$ & 4 & 26 & Complete & MRS 2 \\
\hline 54 & $\mathrm{~F}$ & $\begin{array}{l}\text { Basilar artery } \\
\text { distal occlusion }\end{array}$ & 2 & 40 & $\begin{array}{l}\text { Complete left, } \\
\text { partial right } \mathrm{PCA}^{2}\end{array}$ & MRS 3 \\
\hline 72 & $\mathrm{~F}$ & $\begin{array}{l}\text { MCA }^{\mathrm{a}} \\
100 \% \text { occlusion }\end{array}$ & 5 & 26 & Failed & Died \\
\hline
\end{tabular}

*Internal Carotid Artery: ${ }^{a}$ Middle Cerebral Artery: ${ }^{\circ}$ Anterior Cerebral Artery: ${ }^{2}$ Posterior Cerebral Artery \#National Institute of Health Stroke Scale (Functional grading of stroke size 0 (normal) to 42)

I Modified Rankin Score (0-6) @ 90 days (Functional grading of stroke recovery with 0 normal and 6 dead)

in the other five cases (four terminal ICA occlusions (Figure 3), one distal basilar artery occlusion) and vessel patency of $100 \%$ was obtained. Intra-arterial tPA was given in all cases (total dose $5-15 \mathrm{mg}$ ) in conjunction with the Merci Retriever. One patient died four days post procedure due to a massive stroke, while the outcome of the four successful patients far exceeded the expected prognosis for such large strokes. There were no symptomatic intracerebral hemorrhages.

\section{BRAIN ATTACKS - IMPROVING PATIENT ACCESS TO CATHETER BASEDTHERAPY}

Early reperfusion with catheter based therapy, that is a short door-toballoon time, is the optimal treatment for acute myocardial infarction and it will most likely become the optimal treatment for selected large vessel acute ischemic stroke patients. Without reperfusion therapy these patients have a very poor prognosis. However, very few arrive at hospital in time for possible iv thrombolysis $(<10 \%)$, which when given has limited efficacy in opening large vessel occlusions (<30\%) Mechanical embolectomy has been shown to be safe and effective up to 8 hours after stroke onset, thereby allowing far more severe acute stroke patients the option of revascularization and its proven benefits. Unfortunately there is a severe shortage of neuro-interventionalists in South Africa (approximately 7)(personal communication), and indeed worldwide. In the United States, there are only 385 interventional neuro-radiologists. ${ }^{(13)}$

Clearly in order to provide a 24-hour a day, 365 days per year emergency stroke service a vast increase in the number of skilled neuro-interventionalists is required. It has been proposed that the larger multi-specialty community of carotid interventionalists begin to fill this gap.(13) These specialists (cardiologists, vascular surgeons, 


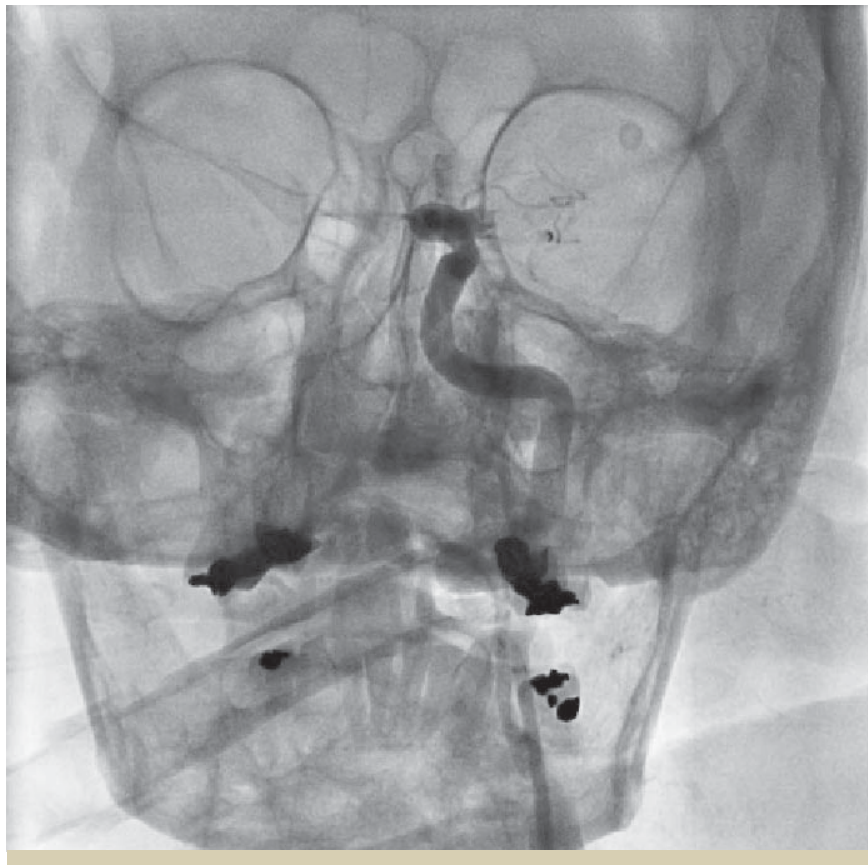

FIGURE 3A: Cerebral angiogram, antero-posterior view, showing complete occlusion of the terminal portion of the left ICA.

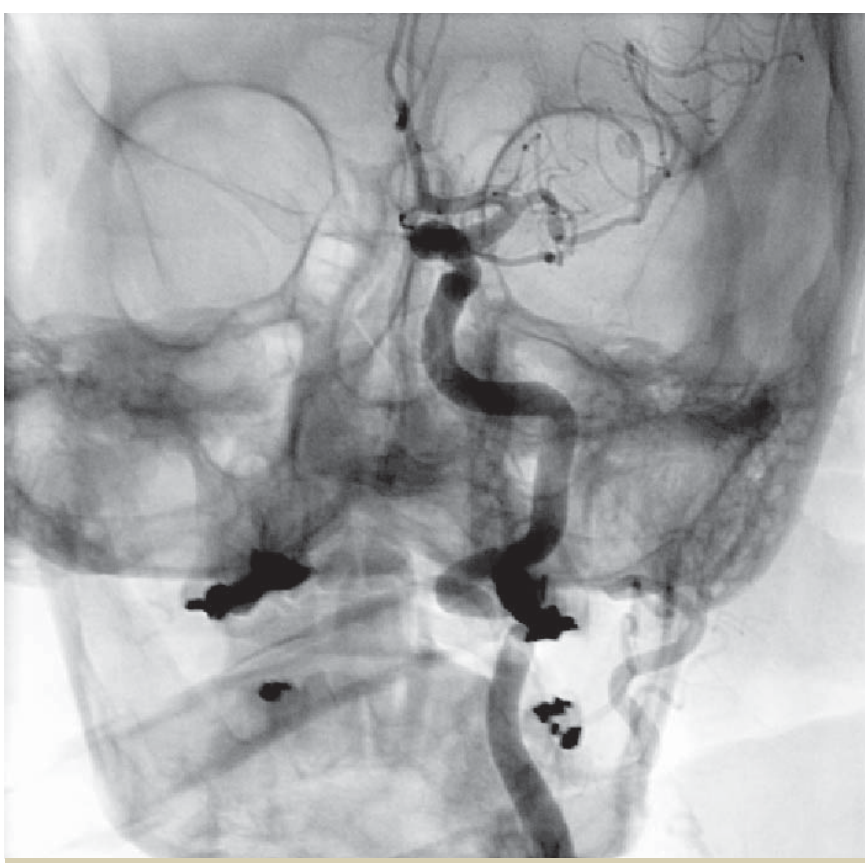

FIGURE 3B: Angiogram showing complete restoration of flow following thrombus extraction.

(Reproduced with permission from Abelson et al. Cardiovascular Journal of Africa, 2008; In press)

radiologists) already have the skills to cannulate the cervical vessels and knowledge of neuro anatomy. It must be emphasized that the interventionalist is merely a member of the emergency stroke team. A multi-disciplinary approach involving the interventionalist, neurologist, emergency unit physicians and radiologists is mandatory to optimize patient outcome in this devastating condition.

\section{REFERENCES:}

I. Declaration Statement, World International Stroke Congress, October 2007, Cape Town, South Africa.

2. The National Institute of Neurological Disorders and Stroke rt-PA Stroke Study Group. Tissue plasminogen activator for acute ischemic stroke. N Engl J Med 1995;333:158|-1587

3. Furlan $A$, et al. Intra-arterial prourokinase for acute ischemic stroke. The PROACT 2 study. JAMA 1999;282:2003-201 I.

4. Combined intravenous and intra-arterial recanalization for acute ischemic stroke. The interventional management of stroke (IMS) study. Stroke 2004;35:904-91 I.

5. Smith WS, Sung G, Starkman S et al. Safety and efficacy of mechanical embolectomy in acute ischemic stroke: results of the Merci trial. Stroke 2005:36: 1432-1438.

6. Marder $\mathrm{V}$ J, et al. Analysis of thrombi retrieved from cerebral arteries of patients with acute ischemic stroke. Stroke 2006:37:2086-2093.

7. Adams HP Jr, et al. Guidelines for the early management of adults with ischemic stroke. Circulation 2007; I I 5:e478-e534.

8. Demaerschalk BM, Yip TR. Economic benefit of increasing utilization of intravenous tissue plasminogen activator for acute ischemic stroke in the United States. Stroke 2005;36:2500-2503

9. Ribo et al. Temporal profile of recanalization after intravenous tissue plasminogen activator. Stroke 2006;37:1000-1004

10. Saqqur M, Uchino K, Demchuk AM, et al. Site of arterial occlusion identified by transcranial doppler predicts the response to intravenous thrombolysis for stroke. Stroke 2007; 38:948-954

II. Smith WS, Sung G, Saver J, et al. Mechanical Thrombectomy for Acute Ischemic Stroke. Final results of the Multi Merci trial. Stroke 2008;39: 1205-1212.

12. Rha JH, Saver $\mathrm{J}$. The impact of recanalization on ischemic stroke outcome: a meta-analysis. Stroke 2007;38:967-973.

13. White CJ, Cates CU, et al. Interventional Stroke Therapy: Current state of the art and needs assessment. Catheterization and Cardiovascular Interventions 2007;70(3): $47 \mid-476$.

14. Kwiatkowski T, Libman R, et al. Effects of Tissue Plasminogen Activator for Acute Ischemic Stroke at One Year. NEJM 1999;340: 1781 - 1787

15. Hacke W, Albers G, et al. The Desmoteplase in Acute Ischemic Stroke Trial: a phase 2 MRI-based 9-hour window acute stroke thrombolysis trial with intravenous desmoteplase. Stroke 2005;36(I):66-73. 